\title{
The relationship between serum concentrations and central nervous system actions of metoprolol
}

\author{
FM GENGO, ${ }^{2}$ JC ERMER,' C CAREY, ${ }^{2}$ GC KALONAROS,' WB McHUGH' \\ From the Dent Neurologic Institute, Millard Fillmore Hospital, Buffalo, New York' and Division of Clinical \\ Pharmacy Sciences, School of Pharmacy, State University of New York at Buffalo', USA
}

SUMMARY The relationship between serum levels of metoprolol, hydroxymetoprolol and changes in psychomotor function as measured by standard reaction time and flash fusion frequency was studied. Blinded subjects were given placebo or $150 \mathrm{mg}$ dose of metoprolol and crossed over on the next study day. Flash fusion frequency, reaction time, and serial blood levels of metoprolol and hydroxymetoprolol were collected. Oral dosing of metoprolol produced no significant changes in reaction time. However, significant decreases in flash fusion frequency were observed from 2 to 6 hours after the dose. Changes in flash fusion frequency were related to levels of metoprolol. Flash fusion frequency changes lagged behind the time course of metoprolol concentrations. The nadir of variance in the metoprolol plus hydroxymetoprolol concentration-effect relationship occurred when potency of hydroxymetoprolol was assumed to be 0.3 of metoprolol. These data suggest that the central nervous system actions of metoprolol are related to metoprolol serum levels and occur at low metoprolol concentrations. However, CNS effects of metoprolol do not intensify at high concentrations. Further CNS activity of hydroxymetoprolol may explain the lag seen in the relationship between concentration and CNS effect.

The increased therapeutic utilisation of the betablockers has been accompanied by an increasing number of reports of CNS activity of these drugs. ${ }^{1}$ Clinical observations of sedation, mental confusion, and psychosis led to studies which have demonstrated diverse neuropharmacologic properties of beta-adrenergic receptor blocking drugs. ${ }^{23}$ There have been several reports documenting impaired cognitive and psychomotor function by betablocker. ${ }^{4-6}$ Despite this increased awareness relatively little is known about the factors that influence the CNS actions of these drugs. ${ }^{7}$ The integration of clinical pharmacokinetic and pharmacodynamic studies can provide some quantification of the intensity and duration of these CNS reactions relative to the prevailing serum drug concentrations.

The purpose of this study was to determine if either the intensity or the duration of the central nervous system actions of a lipid soluble betablocker can be related to the prevailing serum concentrations of drug and/or its metabolite.

Address for reprint requests: Dr Francis $M$ Gengo, Dent Neurologic Institute, Millard Fillmore Hospital, 3 Gates Circle, Buffalo, New York 14209, USA.

Received 3 April 1984. Accepted 21 May 1984

\section{Methods}

\section{Subject selection}

Nine male volunteers between 22 and 32 years of age were examined by a physician and considered to be in good health based on results of routine blood chemistry tests, electrocardiogram, and physical examination.

\section{Study design and procedure}

A double blind random crossover design was used on three nonconsecutive days. Each study day was followed by a two to seven day drug-free interval. Measurements of reaction time and flash fusion frequency were recorded over the course of the control study day and served as baseline control values for each subject. Following "control" day evaluations, volunteers were randomly assigned to receive metoprolol or placebo. Following an overnight fast, volunteers received either a single $150 \mathrm{mg}$ dose of metoprolol or placebo (identical in appearance). Blood samples were drawn from indwelling venous catheters at time 0 , and at $0 \cdot 5,1,2,3,4,6,8$, and 12 hours after the dose of metoprolol or placebo was given. Standardised meals were allowed after 1 hour, 4 hours, and 8 hours. Reaction time and flash fusion frequency were measured at time 0 , and $0 \cdot 5,1,2,3,4,6$, and 8 hours.

\section{Neurophysiologic testing}

Reaction time Reaction time was measured with the subject seated in front of a box with two lights and a button 
switch for each light. As the investigator started a timer, one of the lights on the box was randomly lit. The subject was instructed to press the corresponding button switch as quickly as possible. The timer stopped when the subject pressed the correct button, giving a reaction time in milliseconds. The mean of five separate readings was used as a reaction time.

Flash fusion frequency Subjects viewed flashes of white light monocularly through a Kodak Wratten neutral density gelatin filter $(50 \mathrm{~mm} \times 50 \mathrm{~mm})$, which allowed a $0.1 \%$ transmission of light. Flashes were $5 \mathrm{~ms}$ duration square waves of white light produced by a Grass Photo Stimulator. This device enabled the investigator to vary continuously the rate of the flashes of light from ten to sixty flashes per second. As the rate of flashing was varied, subjects were asked to report when they perceived the flickering light as constant light. The threshold frequency at which a flashing white light was perceived as a constant light was recorded as the fusion frequency. This measurement was performed in a room with constant illumination and was practiced until baseline threshold values were reproducible. We found no difference in the fusion threshold whether the flash frequency was increasing from flashing to constant, or was decreasing from constant to flashing light. ${ }^{8}$

Metoprolol assay Concentrations of metoprolol and hydroxymetoprolol in serum were determined using High Pressure Liquid Chromatography (HPLC)' in conjunction with a fluorescence detector (excitation wavelength of $230 \mathrm{~nm}$, emission wavelength of $300 \mathrm{~nm}$ ). Pronetholol was used as internal standard, on a Waters C18 Microbondapak column. Extraction efficiency for metoprolol and hydroxymetoprolol was $95 \%$ and $97 \%$, respectively. Day-to-day variation was less than $5 \%$ with quantitative sensitivity of $5.0 \mathrm{ng} / \mathrm{ml}$ for both compounds.

Pharmacokinetic/pharmacodynamic calculations Area under the serum concentration time curve (AUC) was determined by La Grange method of area analysis ${ }^{10}$ for each patient. The AUC was then used to determine the apparent oral clearance from the following relationship:

$$
\text { Clearance }=\frac{\text { Dose }}{\mathrm{AUC}_{0-\infty}}
$$

Measurements of pharmacologic effect (FFF or reaction time) and metoprolol serum concentrations were fitted to the "Emax" model as described by Sheiner et al" to obtain estimates of the maximum drug induced effect (Emax) and the serum concentration needed to elicit $50 \%$ of maximal effect (Cp50\%).

$$
\text { Effect }=E \text { (no drug) }-\frac{\text { Emax } \cdot \mathrm{Ct}}{\mathrm{Cp} 50 \%+\mathrm{Ct}}
$$

Where $\mathrm{E}$ (no drug) is the measured effect with no drug present (baseline), $\mathrm{Ct}$ is the measured serum concentration at time t, EMAX is the maximum drug induced effect, and Cp50\% is the serum concentration needed to elicit 50\% Emax. Nonlinear regression analysis was used to fit metoprolol and hydroxymetoprolol concentrations as a function of time according to

$$
C(t)=\sum_{i=1}^{n=1} C_{i} e^{ \pm \lambda} i^{t}
$$

This regression analysis yields estimates of the slopes and intercept values that best fit the time course of metoprolol absorption and disposition. ${ }^{12}$ These estimates are then used in a further non-linear regression analysis to describe the time course of pharmacologic effect in terms of drug concentrations according to

$$
\text { Effect }=E_{(\text {no drug })}-\frac{\text { Emax.keO·Z }}{[C 50 \%+(k e O \cdot Z)]}
$$

where

$$
Z=\sum_{i=1}^{n=1} \frac{C i}{K e O / 2 \cdot 303-\lambda_{i}}\left(e^{ \pm \lambda_{i} \cdot t}-e^{k e O / 2 \cdot 303}{ }^{i t}\right.
$$

where $\mathrm{keO}$ is the rate constant that characterizes the temporal aspects of drug effect."

Metabolite activity The relationship between pharmacologic effect and serum concentration can be determined for parent drug alone or for the total concentration of drug plus the concentration of simultaneously measured metabolite. The contribution of metabolite to the pharmacologic effect can be described by varying the fraction of metabolite concentration, and assessing measured and predicted effect over a range of fractions. Thus, the relative potency (f) of $\alpha$-hydroxymetoprolol to metoprolol in decreasing FFF can be estimated by:

$$
\mathrm{FFF}=\mathrm{FFF}_{(\text {no drug) }}-\frac{\text { Emax } \cdot \mathrm{C} \text { total }}{\mathrm{Cp} 50 \% \pm \mathrm{C} \text { total }}
$$

where

$$
\text { C total }=\mathrm{C} \text { drug }+(\mathrm{f}) \mathrm{C} \text { metabolite }
$$

where FFF is flash fusion frequency, FFF (no drug) is the $T$ measured flash fusion frequency baseline, Emax is th maximal drug induced change, $\mathrm{C}$ drug and $\mathrm{C}$ metabolite are simultaneously measured metoprolol an $\alpha$-hydroxymetoprolol concentrations, and $\mathrm{f}$ is the fractiof $\overrightarrow{0}$ potency of metabolite. The variance of measured and pre $=\stackrel{+}{=}$ dicted response were evaluated at various $f$ values ranging from 0 to 10.0. The nadir of variance is an indicator of the contribution of $\alpha$-hydroxymetoprolol to FFF reduction.

Statistics Unless otherwise indicated all significant relationships are determined using Pearsons correlation coefficient and $p$ values test the hypothesis of a zero population correlation. All significant differences are determined using students $t$ test or ANOVA analysis of variance and $p$ values test the hypothesis that no difference exists.

\section{Results}

Pharmacokinetics As seen in fig 1, metoprolol and $\alpha$-hydroxymetoprolol concentrations displayed an apparent mono-exponential decline and were fitted to a one-compartment pharmacokinetic model. Peak concentrations of metoprolol averaged 277.91 $\pm 25.03 \mathrm{ng} / \mathrm{ml}$, while peak metabolite concentrations averaged $320 \cdot 22 \pm 7 \cdot 18 \mathrm{ng} / \mathrm{ml}$ (mean $\pm \mathrm{SEM}$ ). Metoprolol concentrations peaked at $1.16 \pm 0.49$ hours (mean \pm SD); those of hydroxymetoprolol peaked at $2.39 \pm 2.19$ (mean $\pm \mathrm{SD}$ ). Estimates of 


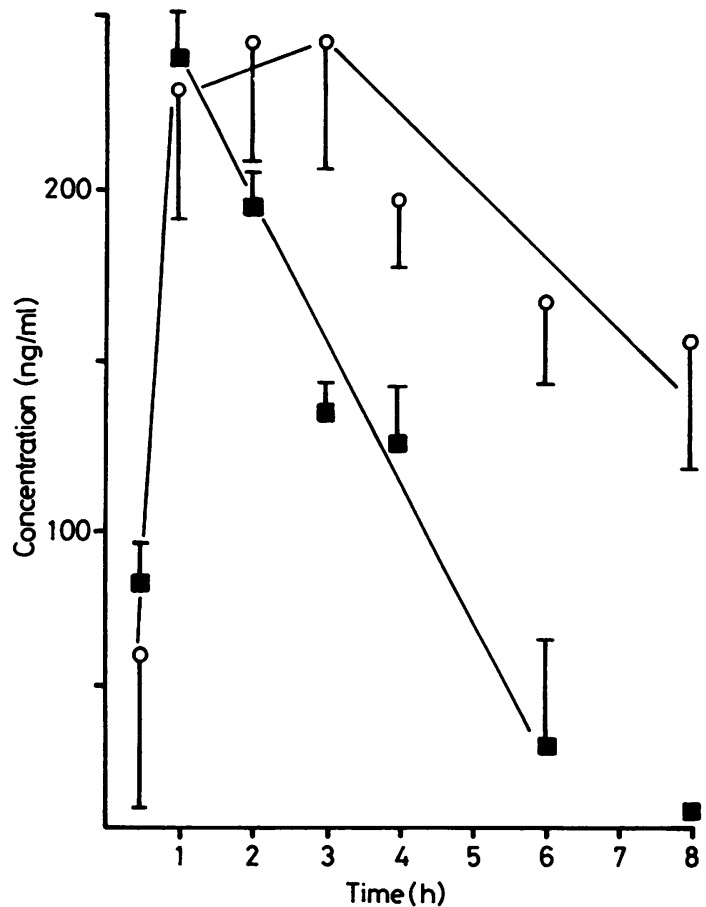

Fig 1 The time course of metoprolol ( $\square$ ) and $\alpha$-hydroxymetoprolol $(O)$ concentrations.

the apparent pharmacokinetic parameters of this analysis are listed in the table. Based on a model independent analysis of area under the curve, equation (1), the apparent oral clearance of metoprolol averaged $261 \cdot 4 \pm 175 \cdot 2 \mathrm{lh}$.

Intensity of pharmacologic effects Reaction times following placebo were essentially the same and those measured during the baseline control day of study. Reaction times after placebo were not significantly different from those measured after the $150 \mathrm{mg}$ single dose of metoprolol. The data from the nine subjects is shown in fig 2 . Flash fusion frequency values measured throughout the baseline control day varied less than $6 \%$ for each subject. Following placebo, FFF measurements were essentially unchanged, with a $7 \%$ maximum variation. Following metoprolol, patients showed a maximum decrease in flash fusion frequency of about $38 \%$. The ratio of treatment FFF to control baseline FFF is seen in fig 2. Metoprolol produced a statistically significant decrease in flash fusion frequency measured at $2,3,4$, and 6 hours as compared to placebo $(p<0.05)$. There was no difference between initial and final FFF values on the metoprolol study day indicating the duration of the drug induced changes in FFF. There was a significant $(p<0.05)$ relationship between metoprolol serum concentrations and the extent of change in FFF. Figure 3 shows changes in FFF fitted with metoprolol serum concentrations using equation (2). Metoprolol concentrations were related to changes in FFF ( $p<0.05-0.01)$ in each subject, as well as in the group data. When all of the metoprolol concentration and FFF data pairs where simultaneously fitted, there was a significant relationship ( $p<0.01, r^{2}=0.84$ ), yielding Emax estimates of $15 \pm 3 \%$, and C50\% $75 \pm 40 \mathrm{ng} / \mathrm{ml}$. Estimates of the individual Emax and $\mathrm{Cp} 50 \%$ values are listed in the table.

Kinetics of pharmacologic effect The maximum change in FFF for the group occurred 3 hours after the dose of metoprolol. Peak metoprolol concentration occurred 1.25 hours after the metoprolol dose. The temporal disparity between serum concentrations and effect can be readily appreciated by comparison of fig 1 and fig 2 . The lag between concentrations and effect averaged $2 \cdot 7$ hours. Following this lag in equilibrium, effect declined in parallel to log serum concentrations. The temporal lag between concentration and effect is quantitatively described by $\mathrm{KeO}$, the rate constant for concentration effect equilibrium. Individual estimates of this parameter

Table Pharmacokinetic and pharmacodynamic parameter estimates

\begin{tabular}{|c|c|c|c|c|c|}
\hline & $\frac{C L o^{*}}{(l / h)}$ & $\frac{\text { Kelt }}{(h-1)}$ & $\frac{E \max }{(\%)}$ & $\frac{C 50 \%}{\text { (ng./ml.) }}$ & $\frac{k e O^{\prime \prime}}{(h-1)}$ \\
\hline $\begin{array}{l}1 \\
2 \\
3 \\
4 \\
5 \\
6 \\
7 \\
8 \\
9\end{array}$ & $\begin{array}{r}49 \\
506 \\
248 \\
233 \\
564 \\
129 \\
302 \\
89 \\
227 \\
261+175\end{array}$ & $\begin{array}{l}0.37 \\
0.36 \\
0.68 \\
0.11 \\
0.72 \\
0.01 \\
0.38 \\
0.21 \\
0.27 \\
0.35+0.23\end{array}$ & $\begin{array}{l}38 \\
16 \\
29 \\
17 \\
23 \\
6 \\
10 \\
10 \\
5 \\
15+39\end{array}$ & $\begin{array}{r}505 \\
34 \\
70 \\
28 \\
28 \\
97 \\
474 \\
10 \\
28 \\
74+40 \text { II }\end{array}$ & $\begin{array}{l}1.61 \\
0.56 \\
3.50 \\
1.60 \\
1.40 \\
1.40 \\
0.94 \\
1.40 \\
0.82 \\
1.43+0.8\end{array}$ \\
\hline
\end{tabular}

Apparent oral clearance.

†Apparent elimination rate constant.

¥Least squares estimate of maximum effect, expressed as percent of baseline

$\$$ Least squares estimate of metoprolol concentration yeilding $50 \%$ of maximal effect.

"Least square estimate of rate constant relating time course of concentration to time course of effect.

INot averaged data. 
are listed in the table. The temporal lag between peak drug concentration and maximum pharmacologic effect was consistent in each subject, and consistent with an active metabolite contributing to the observed drug effect. This possibility was more closely examined by estimating the contributions of metabolite concentrations measured during the time course of pharmacologic effect using equation (3). As seen in fig 4, the nadir in the variance for the relationship between calculated FFF and observed FFF occurs when the relative contribution of $\alpha$-hydroxymetoprolol concentrations are assumed to be 0.3 of the contribution of the metoprolol concentrations. Analysis of variance (ANOVA) and Tukeys test revealed a significant reduction ( $\mathrm{p}<$ 0.01 ) in the variance between calculated and observed FFF when the relative potency of the hydroxymetoprolol is assumed to be $\mathbf{0 \cdot 3 0}$.

\section{Discussion}

Beta-blocker induced reductions in mental alertness have previously been reported. ${ }^{45}$ The data presented in this study demonstrated that the degree of reduction in flash fusion frequency is directly associ-

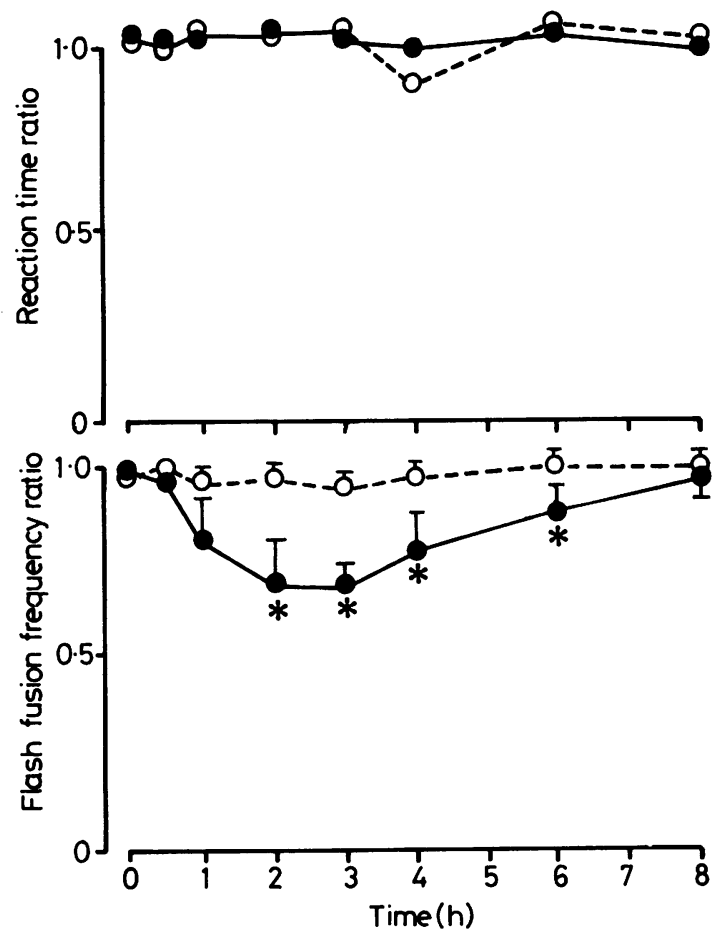

Fig 2 The time course of reaction time and flash fusion frequency following $150 \mathrm{mg}$ of metoprolol (O) or placebo (O). ated with serum concentrations of metoprolol. This relationship was observed consistently within each subject.

There are several important points beyond the finding that changes in flash fusion frequency can be associated with metoprolol concentrations. Metoprolol is a relatively non-protein bound drug that is highly lipid soluble. ${ }^{13}$ These characteristics enable rapid and complete penetration of the blood brain barrier. Cruickshank et al have measured the simultaneous concentrations of metoprolol in serum, CSF and brain tissue, confirming that metoprolol concentrations in the CNS increase readily as serum concentrations increase. ${ }^{14}$ Intuitively it would seem that as serum concentrations of metoprolol increase, and therefore CNS concentrations increase, a proportional increase in the intensity of pharmacologic effect might be expected. However, despite a tenfold increase in metoprolol above concentrations associated with maximal changes in flash fusion frequency, the apparent maximal reduction in flash fusion frequency remained $15 \%$. This suggests that despite high concentrations in the CNS, metoprolol possesses a seemingly low potency in reducing mental alertness. Consistent with this we were not able to demonstrate any difference between metoprolol ando placebo causing reductions in standard reaction time. This is also consistent with several clinicali studies that report a low incidence of CNS side effects with metoprolol, despite its ability to gain access to the CNS. ${ }^{1516}$ This demonstrates thato increasing concentrations of drug at a given effect site do not invariably result in increasing intensity of pharmacologic effect. The intensity of pharmacologic effect is determined by the pharmacodynamic parameters Emax and C50\%, as well as drug concentrations. ${ }^{17}$ It is important to point out the measured pharmacologic effect, and therefore

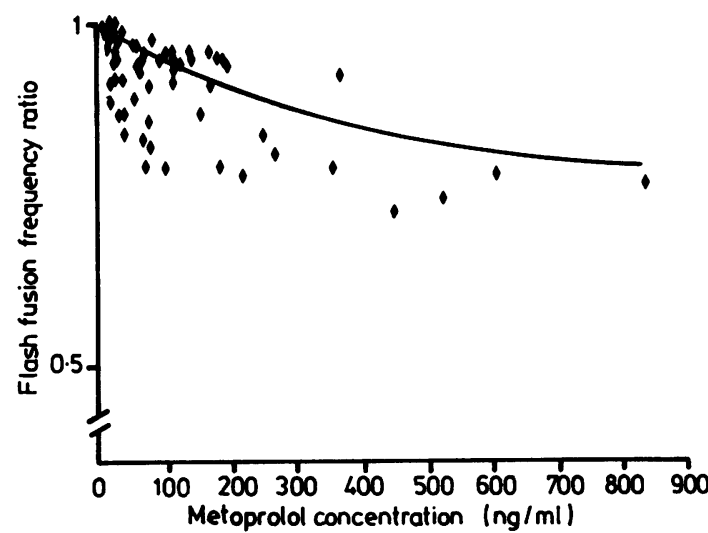

Fig 3 Flash fusion frequency changes at various metoprolol concentrations. 
the pharmacodynamic parameters from clinical studies such as this, are apparent. They likely represent the net effect of several pharmacologic mechanisms. ${ }^{18}$ As such, these parameters do not represent the same parameters as those generated by molecular pharmacologists studying precise pharmacologic mechanisms in specialised preparations.

Another important finding is suggested by the relationship between the time course of metoprolol concentrations and the duration of pharmacologic response. There was a consistent and significant lag between the time of peak drug concentrations and the time of maximal pharmacologic effect. There are several possible explanations for this observation. These explanations can be broadly divided as having either a pharmacokinetic or pharmacodynamic basis. Pharmacokinetic explanations include a lag time in the equilibrium of metoprolol concentrations between the serum and the site of pharmacologic effect. Since the exact sites in the CNS for betablocker induced reductions in FFF are unknown, this explanation cannot be entirely dismissed. This seems unlikely however, since metoprolol has been shown to rapidly gain access to the brain tissue.

A more likely pharmacokinetic explanation for the temporal disparity between the time course of drug concentration and drug effect is the contribution of an active metabolite to the pharmacologic

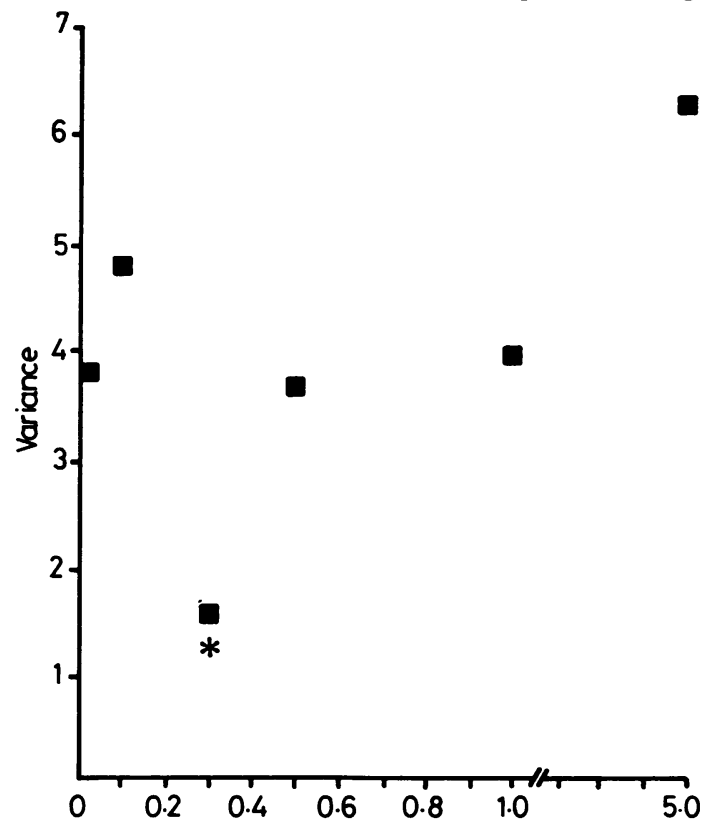

Fig 4 Variance of calculated and observed flash fusion frequency as the assumed fractional potency of hydroxymetoprolol is varied from $0-10$. effect. As metabolite concentrations slowly increased, their contribution to the measured pharmacologic effect would become increasingly more pronounced. This would shift the time of maximum pharmacologic effect to the right, consistent with the time of maximum metoprolol and metabolite concentrations. This is the pattern that appeared in the present study. The possibility that metabolite concentrations contribute to the measured pharmacologic effect was mathematically tested by relating the intensity of effect to the sum of metoprolol plus metabolite concentrations. This is a method previously used by Levy's and Collste ${ }^{20}$ and takes advantage of the large differences between the pharmacokinetics of metoprolol and $\alpha$-hydroxymetoprolol. Variance in the drug concentration:effect relationship is significantly minimised $(p<0.05)$ when the fractional potency of metabolite is between 0.2 and 0.4 . Based on this analysis, $\alpha$-hydroxymetoprolol is $30 \%$ as appears active as metoprolol in reducing mental alertness.

This method is limited since the metabolite is not administered without parent drug. Thus it evaluates metabolite potency in the presence of parent drug. As pointed out by Atkinson et al, ${ }^{21}$ there are many possible reasons why the activity of a metabolite may be different alone rather than when in the presence of the parent drug. However, this method does evaluate the contribution of metabolite to effect under conditions that most resemble the conditions of actual therapy with metoprolol in reducing FFF.

There are pharmacodynamic mechanisms which might also explain the temporal disparity of metoprolol concentrations and effect. It is possible that there was a change in the receptor sensitivity to metoprolol, causing a facilitation of metoprolol binding as time after the dose increasd. Since we do not fully understand the pharmacologic mechanisms of metoprolol in the CNS, we can only mention this as a possibility without further discussion. It is not possible from the data presented to conclude why there is a lag between the metoprolol concentrations and reductions in flash fusion frequency. It is our own feeling that contribution of active metabolite is the most likely explanation. The hydroxymetabolite of metoprolol has been shown to be a weak betablocker. ${ }^{22}$ However, there is no evidence that the CNS actions of metoprolol are mediated by betablockade per se.

In conclusion, the data from the present study demonstrate that there is a significant association between serum concentrations of metoprolol and the intensity of its pharmacologic effect. Despite metoprolol's ability to cross readily the blood brain barrier, it seems to have a relatively weak ability to alter parameters of mental alertness. The maximal 
pharmacological effect is achieved at low concentrations and does not intensify at higher concentrations. It is possible that hydroxymetoprolol contributes to the CNS effects following oral administration of metoprolol.

\section{References}

1 Greenblatt DJ, Koch-Weser J. Adverse reactions to beta-adrenergic receptor blocking drugs. Boston Collaborative Drug Surveillance Group. Drugs. 1974;7:118-29.

2 Neuro-psychiatric effects of adrenergic beta-receptor blocking agents. In: Carlsson $\mathrm{C}$, ed. Advances in Pharmacology, Vol. 12, Berlin, Urban and Schwarzenberg 1976.

${ }^{3}$ Beta-blockers and the Central Nervous System. Kielholz P, ed., Baltimore: University Park Press, 1977.

${ }^{4}$ Landauer AA, Pocock AA. Prott FW. Effects of atenolol and propranolol on human performance and subjective feelings. Psychopharmacology 1979;60:211-5.

${ }^{5}$ Salem SA, McDivitt DG. Central effects of betaadrenergic antagonists. Clin Pharmacol Ther 1983;33:52-7.

- Bryan PC, Efiong DD, Stewart-Jones J, Turner P. Propranolol on tests of visual function and CNS activity. $\mathrm{Br}$ J Clin Pharmacol 1974;1:82-84.

' Koch-Weser J. Non beta-blocking actions of propranolol. New Engl J Med 1975;293:988-9.

${ }^{8}$ Simonson E, Brozek J. Flicker fusion frequency background and applications. Physiol Rev 1952;32:349.

9 Gengo FM, Tokasz M, Kinkel WR. HPLC determination of metoprolol and hydroxymetoprolol in serum, urine, and CSF. J Pharm Sci 1984 (in press).

${ }^{10}$ Caprani O, Sveinsduttir E, Lassen N. SHAM-a method for biexponential curve resolution using initial slope, height, area, and moment of experimental decay curve. J Theor Biol 1975;52:299-315.
" Sheiner LB, Stanski DR, Vozeh S, Miller RO, Ham J Simultaneous modeling of pharmacokinetics and pharmacodynamics: application to d-tubocurarine. Clin Pharmacol Ther 1979;25:358-71.

${ }^{12}$ Metzler CM, Elfring C, McKewen A. A package of computer programs for pharmacokinetic modeling. Biometrics 1974;30:562.

${ }^{13}$ Belpaire F, Bogaert M, Rosseneu M. Binding of betareceptor blocking drugs to human serum albumin to alpha-1-acid glycoprotein, and to human serum. Eur $J$ Clin Pharmacol 1982;22:253-6.

${ }^{14}$ Cruickshank JM, Neil-Dwyer G, Cameron MM, McAinsh K. Beta-receptor blockers and blood brain barrier. Clin Sci 1980;59:4535-55.

is Traford JT, McGonigle R, Bowels J, O' Neal H, Jones D, Halferd $L$. Two year clinical evaluation of atenolol and metoprolol in treatment of hypertension. $\mathrm{Br} J$ Clin Practice 1982:350-52.

${ }^{16}$ Lyngtram O, Ryden L. Metoprolol and atenolol administered once daily for primary hypertension. Acta Med Scand 1981;209:261-6.

${ }^{17}$ Levy G, Gibaldi M, Jusko WJ. Multicompartment pharmacokinetic models and pharmacologic effects. $J$ Pharm Sci 1969;58:422-4.

18 Johnson LC, Chernik D. Sedative-hypnotics and human performance. Phsychopharmacology 1982;76:10113.

${ }^{19}$ Levy G. Kinetics of pharmacologic effect. Clin Phar-T macol Ther 1966;7:362-72.

${ }^{20}$ Collste P, Borg K, Astrum H, Bahr C. Contribution of $4 \stackrel{\mathrm{D}}{\mathrm{D}}$ hydroxy-alprenolol to adrenergic beta-receptor bloc-ه kade of alprenolol. Clin Pharmacol Ther 1979;25:416-22.

${ }^{21}$ Atkinson A, Strong J. Effect of drug metabolites on plasma. J Pharmacok Biopharm 1977;5:95-109.

${ }^{22}$ Regardh C, Ek L, Hoffman K. Plasma levels and betablocking effect of alpha-hydroxymetoprolol, metabolite of metoprolol in the dog. J Pharmacok Biopharm 1979; 7:471-9. 